\title{
Complexity of degenerations of modules
}

\author{
R. Aehle, Ch. Riedtmann and G. Zwara
}

\begin{abstract}
A module $M$ over an associative algebra $A$ over an algebraically closed field $k$ is said to degenerate to a module $N$ if $N$ belongs to the closure of the isomorphism class of $M$ in the algebraic variety of $d$-dimensional $A$-modules, $d \in \mathbb{N}$. We associate a non-negative integer to a degeneration $M \leq_{\operatorname{deg}} N$, its complexity, and study its properties.
\end{abstract}

Mathematics Subject Classification (2000). 14L30, 16 G10.

Keywords. Algebras, modules, degenerations of modules.

\section{Introduction}

Let $k$ be an algebraically closed field, $A$ a finite dimensional associative $k$-algebra with a unit and $\bmod A$ the category of finite dimensional left $A$-modules. Let $\mathbb{M}_{d}(k)$ denote the $k$-algebra of the $d \times d$-matrices with coefficients in $k$. We view $A$ as a quotient of a free associative algebra $k\left\langle X_{1}, \ldots, X_{r}\right\rangle$ by a two-sided ideal $I$. We define the affine variety $\bmod _{A}^{d}(k)$ as the set of $r$-tuples $\left(m_{1}, \ldots, m_{r}\right)$ such that $m_{i} \in \mathbb{M}_{d}(k)$ and $\rho\left(m_{1}, \ldots, m_{r}\right)$ is the zero matrix for any $\rho \in I$. The general linear group $G l_{d}(k)$ acts on $\bmod _{A}^{d}(k)$ by conjugation.

As an ordinary set, $\bmod _{A}^{d}(k)$ is just the set $\operatorname{Hom}_{k-a l g}\left(A, \mathbb{M}_{d}(k)\right)$ and hence $G l_{d}(k)$-orbits in $\bmod _{A}^{d}(k)$ correspond bijectively to isomorphism classes of $d$-dimensional left $A$-modules.

Let $M$ and $N$ be two $d$-dimensional $A$-modules. By definition, $M$ degenerates to $N$, noted $M \leq_{\operatorname{deg}} N$, if $N$ lies in the closure of the $G l_{d}(k)$-orbit of $M$ in $\bmod _{A}^{d}(k)$, with respect to the Zariski topology. This defines a partial order on the set of isomorphism classes of $d$-dimensional $A$-modules.

Denote by $Q$ the quiver

$$
Q=1 \underset{b_{1}}{\stackrel{a_{1}}{\rightleftarrows}} 2 \underset{b_{2}}{\stackrel{a_{2}}{\rightleftarrows}} 3 \cdots
$$

with vertex set $Q_{0}=\mathbb{N} \backslash\{0\}$ and arrows $a_{i}: i \rightarrow i+1, b_{i}: i+1 \rightarrow i$ for every $i \in Q_{0}$. 
We call a representation

$$
T=N_{1} \underset{\beta_{1}}{\stackrel{\alpha_{1}}{\rightleftarrows}} N_{2} \cdots N_{i} \underset{\beta_{i}}{\stackrel{\alpha_{i}}{\rightleftarrows}} N_{i+1} \cdots
$$

of $Q$ in $\bmod A$, the category of finite dimensional $A$-modules, an exact tube if the sequence

$$
0 \rightarrow N_{i} \stackrel{\left(\begin{array}{c}
\beta_{i-1} \\
\alpha_{i}
\end{array}\right)}{\longrightarrow} N_{i-1} \oplus N_{i+1} \stackrel{\left(-\alpha_{i-1}, \beta_{i}\right)}{\longrightarrow} N_{i} \rightarrow 0
$$

or equivalently the square

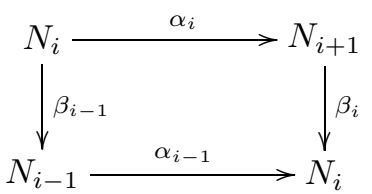

is exact for all $i \geq 1$. Here we set $N_{0}=0$. Note that $N_{i}$ is an $A$-module, that $\alpha_{i}, \beta_{i}$ are $A$-linear and that $\alpha_{i}$ is injective, $\beta_{i}$ is surjective, for all $i \geq 1$. We say that $T$ is an $(M, N)$-tube if there is a natural number $h$ such that

(i) $N_{1} \underset{A}{\stackrel{\sim}{\rightarrow}} N$,

(ii) $N_{h+j+1} \underset{A}{\stackrel{\sim}{\longrightarrow}} N_{h+j} \oplus M$, for all $j \in \mathbb{N}$.

We call the smallest such number $h$ the complexity $\operatorname{cpl}(T)$ of the tube.

Let $T$ be an $(M, N)$-tube. Note that the sequence

$$
0 \rightarrow N_{k} \stackrel{\alpha_{k}}{\longrightarrow} N_{k+1} \stackrel{\beta_{1} \cdots \beta_{k}}{\longrightarrow} N_{1} \rightarrow 0
$$

is exact for any $k$. As $N_{k+1}$ is isomorphic to $N_{k} \oplus M$ for $k \geq \operatorname{cpl}(T)$, there is an exact sequence

$$
0 \rightarrow N_{k} \longrightarrow N_{k} \oplus M \longrightarrow N \rightarrow 0
$$

and therefore $M$ degenerates to $N[5]$.

Conversely, whenever $M$ degenerates to $N$, there exists an $(M, N)$-tube: Indeed, the third author showed in [7] that there is a short exact sequence

$$
0 \rightarrow Z \stackrel{\left(\begin{array}{l}
f \\
g
\end{array}\right)}{\longrightarrow} Z \oplus M \rightarrow N \rightarrow 0
$$

and in [6] he associated an exact tube $T_{f, g}$ with such a sequence (see also Section 4). In fact, $T_{f, g}$ is the cokernel of the injection $\varphi: X \rightarrow X^{\prime}$ between the following representations of $Q$ :

$X:$
$\varphi \mid$
$\downarrow$
$X^{\prime}:$

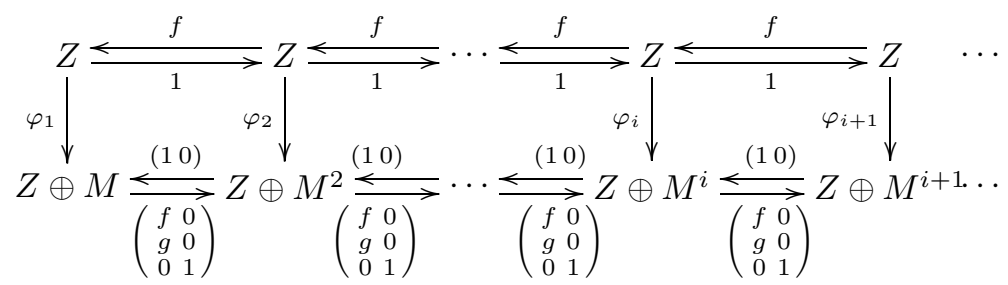


with $\varphi_{i}=\left(f^{i}, g f^{i-1}, \ldots, g\right)^{t}: Z \rightarrow Z \oplus M^{i}$. Both $X$ and $X^{\prime}$ are almost exact tubes: they satisfy all requirements except those related to $a_{1}$ and $b_{1}$. The only condition left to be checked for $T_{f, g}$ is the exactness of the bottom row in the commutative diagram (Figure 1) with exact columns. This is done by diagram chasing.

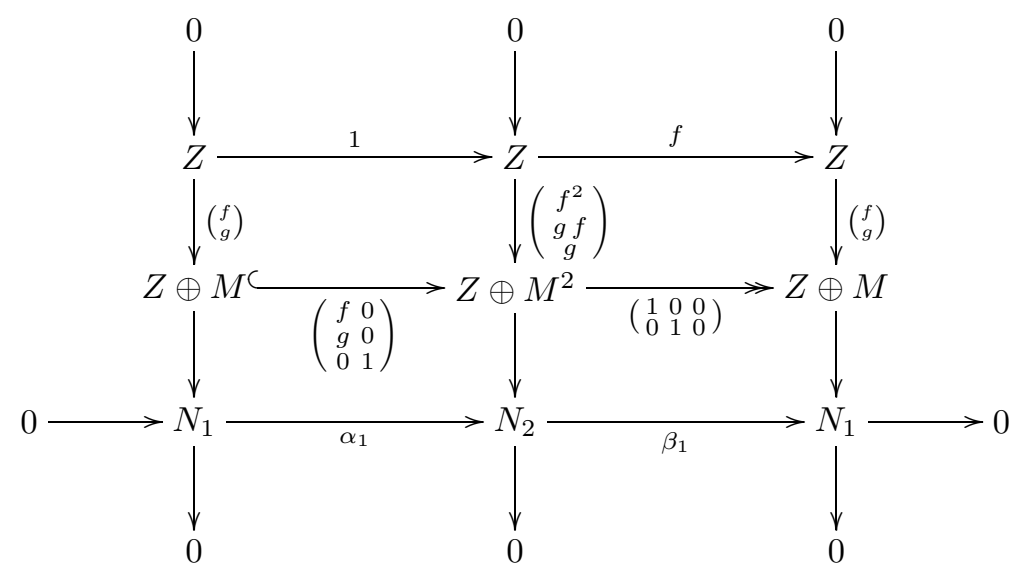

Figure 1

By construction, $N_{1}=\operatorname{coker} \varphi_{1}$ is isomorphic to $N$. Using Fitting's lemma in order to replace $Z$ by a direct summand if necessary in the exact sequence (1.1), we may assume that $f$ is nilpotent, say $f^{h}=0$. Then $\varphi_{h+j}$ has the form $\varphi_{h+j}=$ $\left(0, \ldots, 0, g f^{h-1}, \ldots, g\right)^{t}: Z \rightarrow Z \oplus M^{h+j}$, and its cokernel $N_{h+j}$ is isomorphic to $M^{j} \oplus N_{h}$ for $j \geq 0$. We conclude that $T_{f, g}$ is an $(M, N)$-tube of complexity at most $h$. In fact, $T_{f, g}$ is an $(M, N)$-tube even if $f$ is not nilpotent (compare with Proposition 4.2).

We define the complexity of a degeneration $M \leq_{\operatorname{deg}} N$ to be

$$
\operatorname{cpl}(M, N)=\min \operatorname{cpl}(T),
$$

where $T$ ranges over all $(M, N)$-tubes. This seems to be a good way to measure how "complicated" a degeneration is.

Indeed, we will prove in Sections 3 and 4 that a degeneration $M \leq_{\operatorname{deg}} N$ is of complexity 1 if and only if there exists a non-split exact sequence

$$
0 \rightarrow N^{\prime} \rightarrow M \rightarrow N^{\prime \prime} \rightarrow 0
$$

with $N \stackrel{\sim}{\longrightarrow} N^{\prime} \oplus N^{\prime \prime}$. So these are the "simplest" degenerations. In particular, any degeneration to an indecomposable $N$ must have complexity at least 2 .

It is quite difficult to compute the complexity of a degeneration. The construc- 
tion described before gives an estimate from above: if

$$
0 \rightarrow Z \stackrel{\left(\begin{array}{l}
f \\
g
\end{array}\right)}{\longrightarrow} Z \oplus M \rightarrow N \rightarrow 0
$$

is an exact sequence and $f^{h}=0$, then $\operatorname{cpl}(M, N) \leq h$. Conversely, it is easy to show that

$$
\operatorname{cpl}(M, N) \geq \frac{\ell \ell(M)}{\ell \ell(N)}-1,
$$

where $\ell \ell(X)$ is the Loewy length of $X$; i.e., the smallest number $r$ for which $(\operatorname{rad} A)^{r} \cdot X=0$ (see Proposition 3.5). Both bounds are sharp, but in general the complexity differs from both.

The complexity of a degeneration $M \leq_{\operatorname{deg}} N$ obtained from two degenerations $M \leq_{\operatorname{deg}} P \leq_{\operatorname{deg}} N$ seems to be quite unrelated to the sum of the complexities of $M \leq_{\operatorname{deg}} P$ and $P \leq_{\operatorname{deg}} N$. For instance, if we take non-split exact sequences

$$
0 \rightarrow A_{i} \rightarrow B_{i} \rightarrow C_{i} \rightarrow 0, \quad i=1, \ldots, r,
$$

then there is a sequence of degenerations

$$
\begin{aligned}
\bigoplus_{i=1}^{r} B_{i} & \leq \operatorname{deg}\left(\bigoplus_{i=1}^{r-1} B_{i}\right) \oplus A_{r} \oplus C_{r} \leq_{\operatorname{deg}} \ldots \leq_{\operatorname{deg}}\left(\bigoplus_{i=1}^{s} B_{i}\right) \oplus \bigoplus_{i=s+1}^{r}\left(A_{i} \oplus C_{i}\right) \\
& \leq_{\operatorname{deg}} \ldots \leq_{\operatorname{deg}} \bigoplus_{i=1}^{r}\left(A_{i} \oplus C_{i}\right),
\end{aligned}
$$

but the complexity of

$$
\bigoplus_{i=1}^{r} B_{i} \leq_{\operatorname{deg}} \bigoplus_{i=1}^{r}\left(A_{i} \oplus C_{i}\right)
$$

is 1 . On the other hand, we give an example of a chain of degenerations $M \leq_{\operatorname{deg}}$ $P \leq_{\operatorname{deg}} N$ in Section 5.1 for which $\operatorname{cpl}(M, P)+\operatorname{cpl}(P, N)<\operatorname{cpl}(M, N)$. By Proposition 5.1, a minimal degeneration can have arbitrarily high complexity. A degeneration $M \leq_{\operatorname{deg}} N$ is called minimal if $M$ is not isomorphic to $N$ and moreover $M \leq_{\operatorname{deg}} P \leq_{\operatorname{deg}} N$ implies that $P$ is isomorphic to either $M$ or $N$.

\section{Degenerations, bimodules and exact tubes}

The following construction is explained in detail in [7] (compare also [2] and [3], pp. 176-177): If $M \leq_{\operatorname{deg}} N$ is a degeneration, there exists a discrete valuation $k$-algebra $R$ with maximal ideal $\mathfrak{m}$ and residue class field $k$ and an $A$ - $R$-bimodule $\mathcal{Y}$, which is free of rank $d$ over $R$, such that

i) $\mathcal{Y} / \mathfrak{m} \cdot \mathcal{Y} \underset{A}{\stackrel{\sim}{\longrightarrow}} N$

ii) $\mathcal{Y}$ contains $R \otimes_{k} M$ as an $A$ - $R$-submodule. 
These data are related to mapping a curve $c$ to $\bmod _{A}^{d}(k)$ in such a way that its image lies generically in the orbit of $M$ and intersects the orbit of $N$. Assuming $c$ to be non-singular and passing to the completion, we may assume that $R=k[[t]]$. The representation $T=\left(N_{i}, \alpha_{i}, \beta_{i}\right)$ defined by the setting

$$
N_{i}=\mathcal{Y} /\left(t^{i}\right) \cdot \mathcal{Y}
$$

and letting $\alpha_{i}: N_{i} \rightarrow N_{i+1}$ and $\beta_{i}: N_{i+1} \rightarrow N_{i}$ be induced by multiplication by $t$ and the identity, respectively, is easily seen to be an exact tube, and by [7] it is moreover an $(M, N)$-tube.

This construction associating an exact tube with a bimodule is an equivalence:

Proposition 2.1. The category $\mathcal{T}$ of exact tubes is equivalent to the category $\bmod f A-k[[t]]$ of $A-k[[t]]$-bimodules which are free of finite rank over $k[[t]]$.

Proof. We just describe a quasi-inverse functor. For an exact tube $T=\left(N_{i}, \alpha_{i}, \beta_{i}\right)$ we set

$$
\mathcal{Y}=\lim \left(N_{i}, \beta_{i}\right)
$$

and we put

$$
t \cdot\left(n_{1}, n_{2}, \ldots\right)=\left(0, \alpha_{1}\left(n_{1}\right), \alpha_{2}\left(n_{2}\right), \ldots\right)
$$

for any infinite sequence $\left(n_{1}, n_{2}, \ldots\right)$ with $n_{i} \in N_{i}$ and $\beta_{i}\left(n_{i}\right)=n_{i-1}$ representing an element of $\mathcal{Y}$. As $T$ is an exact tube, this defines an $A-k[[t]]$-bimodule structure on $\mathcal{Y}$. As $t$ acts without torsion, $\mathcal{Y}$ is free as a $k[[t]]$-module, and its rank equals $\operatorname{dim}_{k} N_{1}$, since clearly $\mathcal{Y} /(t) \cdot \mathcal{Y}$ is isomorphic to $N_{1}$.

We give a direct construction of the bimodule corresponding to $T_{f, g}$ for an exact sequence

$$
0 \rightarrow Z \stackrel{\left(\begin{array}{l}
f \\
g
\end{array}\right)}{\longrightarrow} Z \oplus M \rightarrow N \rightarrow 0
$$

with a nilpotent map $f$. Set

$$
\mathcal{Y}_{f, g}=k[[t]] \otimes_{k} M \oplus Z
$$

as an $A$-module and define the action of $t$ on $Z$ by

$$
t \cdot(0, z)=(1 \otimes g(z), f(z)) .
$$

Clearly, this action of $t$ is torsion free, and $\mathcal{Y}_{f, g} /(t) \mathcal{Y}_{f, g}$ is isomorphic to $N$, so that $\mathcal{Y}_{f, g}$ actually belongs to $\bmod f A-k[[t]]$. It is easy to see that the exact tube associated with $\mathcal{Y}_{f, g}$ is $T_{f, g}$.

We will need the following truncated version of an exact tube: 
Definition 2.2. For $m \geq 1$, an exact tube of height $m$ is a representation in $\bmod A$

$$
N_{1} \underset{\beta_{1}}{\stackrel{\alpha_{1}}{\rightleftarrows}} N_{2} \ldots N_{m-1} \underset{\beta_{m-1}}{\stackrel{\alpha_{m-1}}{\rightleftarrows}} N_{m}
$$

of the full subquiver $Q_{m}$ of $Q$ whose vertices are $1,2, \ldots, m$, such that the square

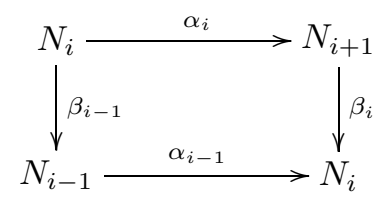

is exact for $i=1, \ldots m-1$. Again we set $N_{0}=0$.

The category of exact tubes of height $m$ is equivalent to the category of $A$ $k[t] /\left(t^{m}\right)$-bimodules which are free of finite rank over $k[t] /\left(t^{m}\right)$.

Obviously, an exact tube $T$ restricts to an exact tube $T_{\leq m}$ of height $m$ for all $m$. We will see in Section 4 that an $M$-extendible tube $T \stackrel{=}{=}\left(N_{i}, \alpha_{i}, \beta_{i}\right)$ of height $h \geq 1$ (see next definition) is always the restriction of an $\left(M, N_{1}\right)$-tube.

Definition 2.3. A tube $T=\left(N_{i}, \alpha_{i}, \beta_{i}\right)$ of height $h$ is called $M$-extendible if there is a decomposition $N_{h}=Z \oplus Z^{\prime}$ and an exact sequence

$$
0 \rightarrow Z \stackrel{\left(\begin{array}{l}
a \\
b
\end{array}\right)}{\longrightarrow} N_{h-1} \oplus M \stackrel{(c d)}{\longrightarrow} Z^{\prime} \rightarrow 0
$$

such that $a=\left.\beta_{h-1}\right|_{Z}$ and $c=p r_{Z^{\prime}} \circ \alpha_{h-1}$, where $p r_{Z^{\prime}}: Z \oplus Z^{\prime} \rightarrow Z^{\prime}$ is the natural projection.

We end this section with some questions. We do not know how to describe the full subcategory of $\bmod f A-k[[t]]$ corresponding to $(M, N)$-tubes. Conceivably, its objects are just those bimodules $\mathcal{Y}$ which contain $k[[t]] \otimes_{k} M$ as a subbimodule. This would follow if we knew that any $(M, N)$-tube is of the form $\mathcal{Y}_{f, g}$ for some exact sequence $(2.1)$.

\section{Complexity}

Definition 3.1. We call a map

$$
\left(\begin{array}{l}
f \\
g
\end{array}\right): Z \rightarrow Z \oplus M
$$

an $(M, N)$-monomorphism provided $N$ is isomorphic to coker $\left(\begin{array}{l}f \\ g\end{array}\right)$. 
Recall that, for a degeneration $M \leq_{\operatorname{deg}} N$, we defined the complexity as

$$
\operatorname{cpl}(M, N)=\min \operatorname{cpl}(T),
$$

where $T$ ranges over all $(M, N)$-tubes. There always are $(M, N)$-tubes with different complexities. For instance, if $(f, g)^{t}: Z \rightarrow Z \oplus M$ is an $(M, N)$-monomorphism and we set

$$
f^{\prime}=\left(\begin{array}{ll}
0 & 1 \\
f & 0
\end{array}\right): Z^{2} \longrightarrow Z^{2}, \quad g^{\prime}=(g 0): Z^{2} \longrightarrow M
$$

the map $\left(f^{\prime}, g^{\prime}\right)^{t}$ will be an $(M, N)$-monomorphism, too, and it is easy to see that

$$
\operatorname{cpl}\left(T_{f^{\prime}, g^{\prime}}\right)=2 \operatorname{cpl}\left(T_{f, g}\right) .
$$

Theorem 3.2. Let $h \geq 1$ be a natural number and $M \leq_{\operatorname{deg}} N$ a degeneration. The following conditions are equivalent:

(i) $\operatorname{cpl}(M, N) \leq h$

(ii) There is an exact sequence

$$
0 \longrightarrow Z \stackrel{\left(\begin{array}{l}
f \\
g
\end{array}\right)}{\longrightarrow} Z \oplus M \longrightarrow N \longrightarrow 0
$$

such that $\operatorname{cpl}\left(T_{f, g}\right) \leq h$.

(iii) There exists an exact tube $T=\left(N_{i}, \alpha_{i}, \beta_{i}\right)$ of height $2 h+1$ with $N \underset{A}{\stackrel{\sim}{\longrightarrow}} N_{1}$ and such that

$$
N_{h+j+1} \underset{A}{\stackrel{\sim}{\longrightarrow}} N_{h+j} \oplus M
$$

for $j=0, \ldots, h$.

(iv) There exists an $M$-extendible exact tube $T=\left(N_{i}, \alpha_{i}, \beta_{i}\right)$ of height $h$ with $N \underset{A}{\stackrel{\sim}{\longrightarrow}} N_{1}$

Proof. Most ingredients for the proof will be given in Section 4. Here we indicate how they fit together: The implications (ii) $\Rightarrow$ (i) $\Rightarrow$ (iii) are obvious. The results of Section 4 up to Proposition 4.6 give that (ii) implies (iv), and Proposition 4.8 shows (iv) $\Rightarrow$ (ii). Finally, the implication (iii) $\Rightarrow$ (ii) follows from Proposition 4.9 and the next lemma.

Lemma 3.3. Let $T=\left(N_{i}, \alpha_{i}, \beta_{i}\right)$ be an $(M, N)$-tube, and assume that $N_{h+1} \stackrel{\sim}{\rightarrow}$ $N_{h} \oplus M$ for some $h \geq 1$. Then $\operatorname{cpl}(T) \leq h$.

Proof. As $T$ is an $(M, N)$-tube, there exists a natural number $j \geq h$ such that $N_{i+1} \stackrel{\sim}{\rightarrow} N_{i} \oplus M$ for all $i \geq j$. Take an integer $i$ with $h<i<j$, and consider the 
two exact squares

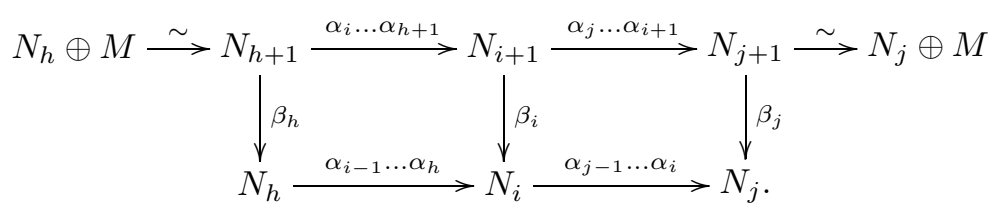

The big square splits, and therefore the two small squares split as well. We conclude that $N_{i+1}$ is isomorphic to $N_{i} \oplus M$.

As $N_{0}=0$, our theorem takes the following simpler form for $h=1,2$ :

Corollary 3.4. Let $M \leq_{\operatorname{deg}} N$ be a degeneration. Then

i) $\operatorname{cpl}(M, N) \leq 1$ if and only if $N=Z \oplus Z^{\prime}$ and there exists an exact sequence

$$
0 \rightarrow Z \rightarrow M \rightarrow Z^{\prime} \rightarrow 0 .
$$

ii) $\operatorname{cpl}(M, N) \leq 2$ if and only if there exist two exact squares
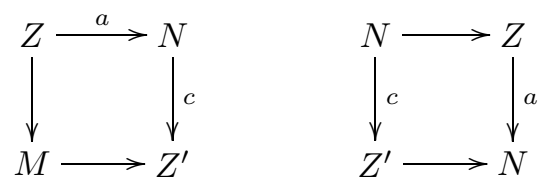

Proposition 3.5. For any degeneration $M \leq_{\operatorname{deg}} N$ we have

$$
\operatorname{cpl}(M, N) \geq \frac{\ell \ell(M)}{\ell \ell(N)}-1,
$$

where $\ell \ell(X)$ denotes the Loewy length of $X$; i.e., the smallest integer $r$ such that $(\operatorname{rad} A)^{r} X=0$.

Proof. Choose an $(M, N)$-tube $T=\left(N_{i}, \alpha_{i}, \beta_{i}\right)$ of complexity $h=\operatorname{cpl}(M, N)$. Then $M$ is a direct summand of $N_{h+1}$, and hence $\ell \ell(M) \leq \ell \ell\left(N_{h+1}\right)$. We claim that, for all $i \geq 1$,

$$
\ell \ell\left(N_{i}\right) \leq i \ell \ell\left(N_{1}\right)
$$

In fact, for any exact sequence

$$
0 \rightarrow A \rightarrow B \rightarrow C \rightarrow 0
$$

the relation

$$
\ell \ell(B) \leq \ell \ell(A)+\ell \ell(C)
$$

holds true. Our claim follows by induction, considering the exact sequences

$$
0 \rightarrow N_{i-1} \rightarrow N_{i} \rightarrow N_{1} \rightarrow 0 .
$$




\section{Exact tubes from monomorphisms}

Throughout this section, $(f, g)^{t}: Z \rightarrow Z \oplus M$ denotes an $(M, N)$-monomorphism.

Definition 4.1. We call two exact tubes $T=\left(N_{i}, \alpha_{i}, \beta_{i}\right)$ and $T^{\prime}=\left(N_{i}^{\prime}, \alpha_{i}^{\prime}, \beta_{i}^{\prime}\right)$ similar if $N_{i}$ is isomorphic to $N_{i}^{\prime}$ for all $i \geq 1$.

So we do not ask for any compatibility with the maps in the tubes. Note that the property of being an $(M, N)$-tube is preserved under similarity, and so is complexity.

Proposition 4.2. There is a direct summand $Z^{\prime}$ of $Z$ and an exact sequence

$$
0 \rightarrow Z^{\prime} \stackrel{\left(\begin{array}{l}
\left.f\right|_{Z^{\prime}} \\
\left.g\right|_{Z^{\prime}}
\end{array}\right)}{\longrightarrow} Z^{\prime} \oplus M \rightarrow N \rightarrow 0
$$

such that $\left.f\right|_{Z^{\prime}}$ is nilpotent and $T_{f, g}$ is similar to $T_{\left.f\right|_{Z^{\prime}},\left.g\right|_{Z^{\prime}}}$. As a consequence, $T_{f, g}$ is an $(M, N)$-tube.

Proof. By Fitting's lemma, there is a decomposition $Z=Z^{\prime} \oplus Z^{\prime \prime}$ of $Z$ as a direct sum which is preserved under $f$ and such that $f^{\prime}=\left.f\right|_{Z^{\prime}}$ is nilpotent and $f^{\prime \prime}=\left.f\right|_{Z^{\prime \prime}}$ is an automorphism of $Z^{\prime \prime}$. Set $g^{\prime}=\left.g\right|_{Z^{\prime}}$ and $g^{\prime \prime}=\left.g\right|_{Z^{\prime \prime}}$. Obviously the maps

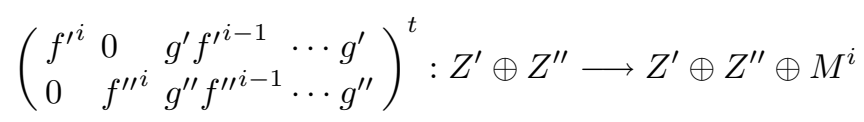

and

$$
\left(f^{\prime i} \quad g^{\prime} f^{\prime i-1} \cdots g^{\prime}\right)^{t}: Z^{\prime} \longrightarrow Z^{\prime} \oplus M^{i}
$$

have isomorphic cokernels as $\left(f^{\prime \prime}\right)^{i}$ is an isomorphism for $i \geq 1$. Since $f^{\prime}$ is nilpotent, $T_{f^{\prime}, g^{\prime}}$ is an $(M, N)$-tube.

Remark 4.3. Suppose that $f^{h}=0$. As

$$
\varphi_{h+j}=\left(0, \ldots, 0, g f^{h-1}, \ldots, g\right)^{t}: Z \longrightarrow Z \oplus M^{h+j},
$$

for $j \in \mathbb{N}$, the exact tube $T_{f, g}$ has the following particularly simple form:

$$
\begin{aligned}
& N_{h+j}=Z \oplus M^{j} \oplus Z^{\prime}, \quad N_{h+j+1}=Z \oplus M^{j+1} \oplus Z^{\prime}, \\
& \alpha_{h+j}=\left(\begin{array}{ll}
f & 0 \\
g & 0 \\
0 & 1
\end{array}\right): Z \oplus\left(M^{j} \oplus Z^{\prime}\right) \rightarrow Z \oplus M \oplus\left(M^{j} \oplus Z^{\prime}\right) \text {, } \\
& \beta_{h+j}=\left(\begin{array}{ccc}
1 & 0 & 0 \\
0 & k & l
\end{array}\right):\left(Z \oplus M^{j}\right) \oplus M \oplus Z^{\prime} \rightarrow\left(Z \oplus M^{j}\right) \oplus Z^{\prime},
\end{aligned}
$$

for $j \in \mathbb{N}$, where $Z^{\prime}$ is a cokernel of

$$
\psi=\left(g \circ\left(f^{h-1}, \ldots, f, 1\right)\right)^{t}: Z \longrightarrow M^{h}
$$

and

$$
(k, l): M \oplus Z^{\prime} \longrightarrow Z^{\prime}
$$


is obtained from the commutative diagram

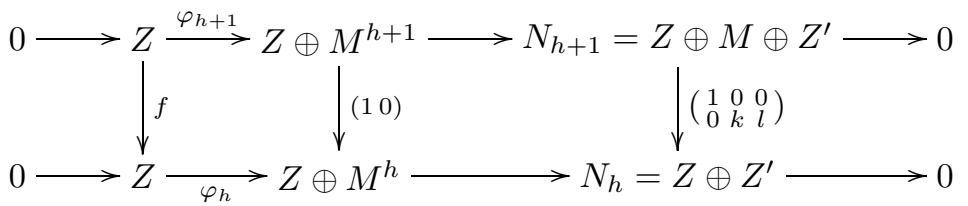

with exact rows.

Our next goal is to show that, up to similarity, we may choose $g \in \operatorname{rad}(Z, M)$. We start with an auxiliary result:

Lemma 4.4. The tube $T_{f, g}$ is similar to $T_{f^{\prime}, g}$ with $f^{\prime}=f-h g$, where $h: M \rightarrow Z$ is any homomorphism.

Proof. It suffices to check the identity $\psi_{i} \circ \varphi_{i}^{\prime}=\varphi_{i}$, for $i \geq 1$, where

$$
\begin{aligned}
\varphi_{i} & =\left(f^{i}, g f^{i-1}, \ldots, g\right)^{t}: Z \longrightarrow Z \oplus M^{i}, \\
\varphi_{i}^{\prime} & =\left(f^{\prime \prime}, g f^{\prime i-1}, \ldots, g\right)^{t}: Z \longrightarrow Z \oplus M^{i}
\end{aligned}
$$

and

$$
\psi_{i}:=\left(\begin{array}{cccccc}
1 & h & f h & f^{2} h & \cdots & f^{i-1} h \\
0 & 1 & g h & g f h & \cdots & g f^{i-2} h \\
0 & 0 & 1 & g h & \ddots & \vdots \\
\vdots & \ddots & \ddots & \ddots & \ddots & g f h \\
\vdots & & \ddots & \ddots & \ddots & g h \\
0 & \cdots & \cdots & \cdots & 0 & 1
\end{array}\right): Z \oplus M^{i} \rightarrow Z \oplus M^{i} .
$$

The key is the equation

$$
f^{r}=f^{\prime r}+\sum_{s=0}^{r-1} f^{s}(h g) f^{\prime r-1-s}, \quad r \geq 1,
$$

which is proved by induction.

Proposition 4.5. There exists a direct summand $Z^{\prime}$ of $Z$ and an exact sequence

$$
0 \rightarrow Z^{\prime} \stackrel{\left(\begin{array}{l}
f^{\prime} \\
g^{\prime}
\end{array}\right)}{\longrightarrow} Z^{\prime} \oplus M \rightarrow N \rightarrow 0
$$

with $g^{\prime} \in \operatorname{rad}\left(Z^{\prime}, M\right)$ and such that $T_{f, g}$ is similar to $T_{f^{\prime}, g^{\prime}}$.

Proof. If $g \in \operatorname{rad}(Z, M)$, there is nothing to be proved. Otherwise, we prove that a sequence (4.1) exists such that $T_{f, g}$ is similar to $T_{f^{\prime}, g^{\prime}}$ and $\operatorname{dim} Z^{\prime}<\operatorname{dim} Z$ and then proceed by induction on $\operatorname{dim} Z$. We choose a non-zero direct summand $Z_{2}$ of $Z$ for which $\left.g\right|_{Z_{2}}$ is a section. Replacing $Z$ by an isomorphic module if 
necessary, which leads to an isomorphic tube, we may assume that $Z=Z_{1} \oplus Z_{2}$, $M=M_{1} \oplus Z_{2}$

$$
g=\left(\begin{array}{ll}
q & 0 \\
0 & 1
\end{array}\right) \text { and } f=\left(\begin{array}{ll}
a & c \\
b & d
\end{array}\right) .
$$

Applying the preceding lemma for

$$
h=\left(\begin{array}{ll}
0 & c \\
0 & d
\end{array}\right)
$$

we obtain a monomorphism $\left(\begin{array}{c}f^{\prime \prime} \\ g\end{array}\right)$ of the form

$$
\left(\begin{array}{c}
f^{\prime \prime} \\
g
\end{array}\right)=\left(\begin{array}{ll}
a & 0 \\
b & 0 \\
q & 0 \\
0 & 1
\end{array}\right): Z_{1} \oplus Z_{2} \rightarrow Z_{1} \oplus Z_{2} \oplus M_{1} \oplus Z_{2}
$$

Now we may take $Z^{\prime}=Z_{1}, M=Z_{2} \oplus M_{1}, f^{\prime}=a$ and $g^{\prime}=\left(\begin{array}{l}b \\ q\end{array}\right)$.

Proposition 4.6. Set $h=\operatorname{cpl}\left(T_{f, g}\right)$, and suppose that $g \in \operatorname{rad}(Z, M)$ and that $f$ is nilpotent. Then $\left(T_{f, g}\right)_{\leq h}$ is $M$-extendible.

Proof. Our assumptions on $f$ and $g$ imply that, for some $i$, the restriction $\left.\psi\right|_{Z}$ of the composition

$$
\psi=\left(\begin{array}{cc}
\varphi_{i} & 0 \\
0 & 1_{M^{h}}
\end{array}\right): Z \oplus M^{h} \rightarrow Z \oplus M^{i} \oplus M^{h}
$$

of the maps

$$
Z \oplus M^{h} \stackrel{\left(\begin{array}{ll}
f & 0 \\
g & 0 \\
0 & 1
\end{array}\right)}{\longrightarrow} Z \oplus M^{1+h} \rightarrow \cdots \stackrel{\left(\begin{array}{ll}
f & 0 \\
g & 0 \\
0 & 1
\end{array}\right)}{\longrightarrow} Z \oplus M^{i+h}
$$

belongs to $\operatorname{rad}\left(Z, Z \oplus M^{i+h}\right)$. By construction of $T_{f, g}$, the square

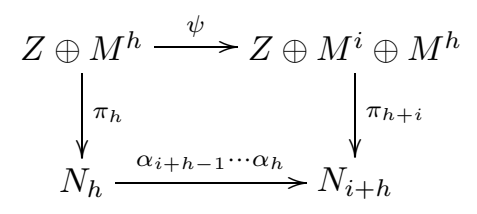

is exact, where $\pi_{j}: Z \oplus M^{j} \rightarrow N_{j}$ is the projection to the cokernel of $\varphi_{j}: Z \rightarrow$ $Z \oplus M^{j}$, and it splits, since $h=\operatorname{cpl}\left(T_{f, g}\right)$. Therefore, $\left.\pi_{h}\right|_{Z}$ is a section, and replacing $N_{h}$ by an isomorphic module, we may assume that

$$
N_{h}=Z \oplus Z^{\prime}, \quad \pi_{h}=\left(\begin{array}{ll}
1 & * * \\
0 & d *
\end{array}\right): Z \oplus M \oplus M^{h-1} \longrightarrow Z \oplus Z^{\prime},
$$

where $*$ is an arbitrary map. 
Now consider the exact squares

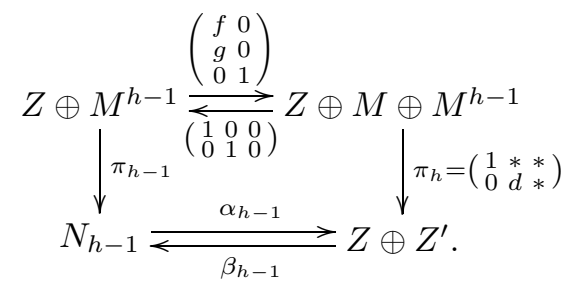

It is easy to see that the square

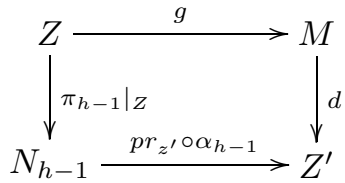

is exact as well. Moreover, we have

$$
\left.\pi_{h-1}\right|_{Z}=\left.\beta_{h-1}\right|_{Z}
$$

Next we recall a different construction for $T_{f, g}$, which has been presented for the most part in [6]. From $(f, g)^{t}$ we obtain the commutative diagram (Figure 2) with exact rows and $\left(k_{i}, l_{i}\right)=\beta_{i}\left(k_{i+1}, l_{i+1}\right)$ for $i \leq m-1$.

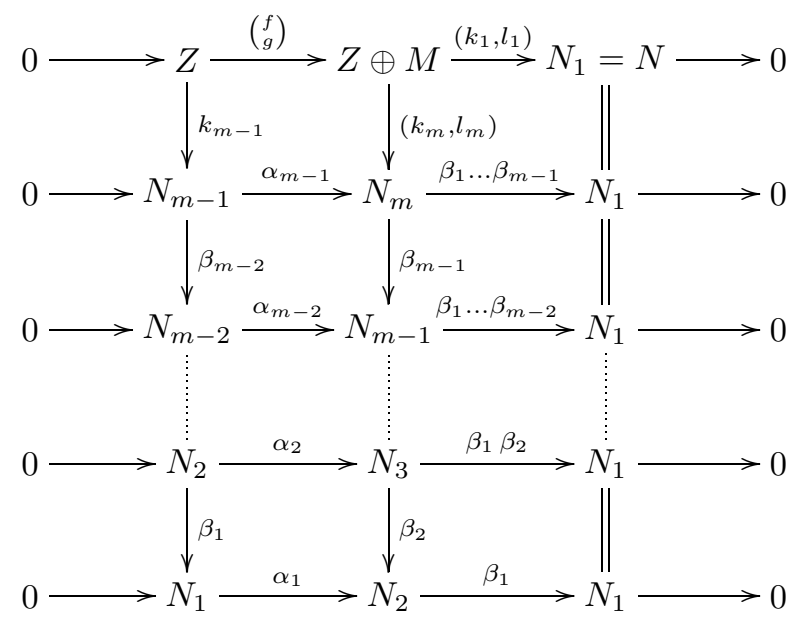

Figure 2

The next step is always obtained by squeezing the push-out of the top sequence by $k_{m}$ between the two top rows. 
We claim that the exact tube $\left(N_{i}, \alpha_{i}, \beta_{i}\right)$ of height $m$ thus obtained is isomorphic to the restriction $\left(T_{f, g}\right)_{\leq m}$ of $T_{f, g}$.

By induction, we obtain the following series of exact squares:

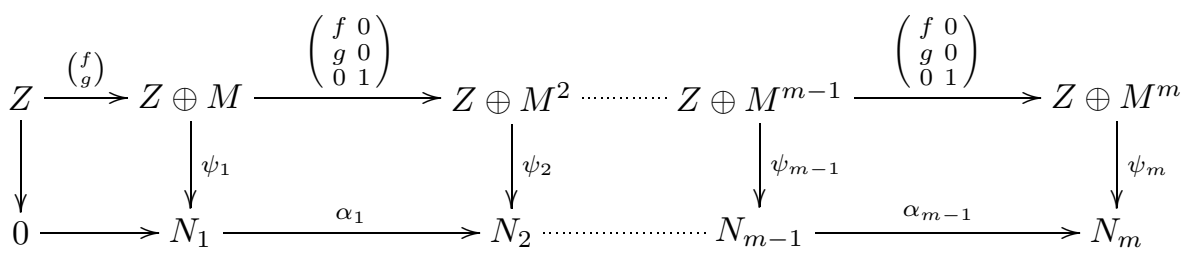

with $\psi_{i}=\left(k_{i}, l_{i}, \alpha_{i-1} l_{i-1}, \ldots, \alpha_{i-1} \ldots \alpha_{1} l_{1}\right): Z \oplus M^{i} \rightarrow N_{i}$.

Note that the composition of the first $i$ maps of the top row is just $\varphi_{i}: Z \rightarrow$ $Z \oplus M^{i}$ and that the sequence

$$
0 \rightarrow Z \stackrel{\varphi_{i}}{\longrightarrow} Z \oplus M^{i} \stackrel{\psi_{i}}{\longrightarrow} N_{i} \rightarrow 0
$$

is exact for $i=1, \ldots, m$. So $N_{i} \stackrel{\sim}{\rightarrow} \operatorname{coker} \varphi_{i}$, and the maps $\alpha_{i}$ are the ones we claim. As for $\beta_{i}$, it suffices to show that

$$
\psi_{i} \circ\left(\begin{array}{ll}
1 & 0
\end{array}\right)=\beta_{i} \circ \psi_{i+1} .
$$

This follows easily from the explicit formulas for $\psi_{i}, \psi_{i+1}$, the equation

$$
\left(k_{i}, l_{i}\right)=\beta_{i}\left(k_{i+1}, l_{i+1}\right)
$$

and the fact that $\left(N_{i}, \alpha_{i}, \beta_{i}\right)$ is an exact tube of height $m$. As a consequence we have:

Remark 4.7. Let $(f, g)^{t}$ be an $(M, N)$-monomorphism and $T^{\prime}=\left(N_{i}^{\prime}, \alpha_{i}^{\prime}, \beta_{i}^{\prime}\right)$ an exact tube of height $m$. Then $T^{\prime}$ is isomorphic to $\left(T_{f, g}\right)_{\leq m}$ if and only if there exists an exact square

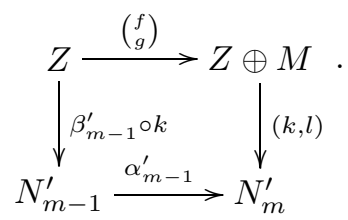

Proposition 4.8. Any $M$-extendible exact tube $T=\left(N_{i}, \alpha_{i}, \beta_{i}\right)$ of height $m$ with $N_{1} \underset{A}{\stackrel{\sim}{\longrightarrow}} N$ is the restriction of the exact tube $T_{f, g}$ to $Q_{m}$ for some $(M, N)$ monomorphism $(f, g)^{t}$.

Proof. Let

$$
0 \rightarrow Z \stackrel{\left(\begin{array}{l}
a \\
b
\end{array}\right)}{\longrightarrow} N_{m-1} \oplus M \stackrel{(c d)}{\longrightarrow} Z^{\prime} \rightarrow 0
$$


be an exact sequence with $N_{m}=Z \oplus Z^{\prime}, a=\left.\beta_{m-1}\right|_{Z}$ and $c=\operatorname{pr}_{Z^{\prime}} \circ \alpha_{m-1}$. The square

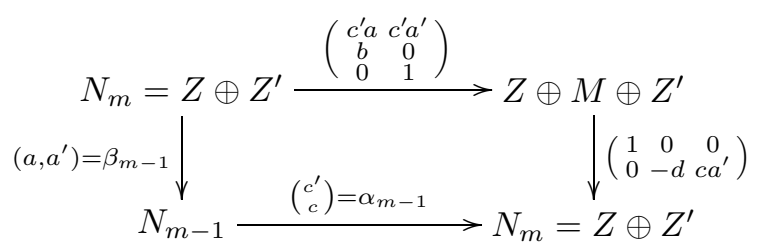

is exact. Setting

$$
N_{m+1}=Z \oplus M \oplus Z^{\prime}, \quad \alpha_{m}=\left(\begin{array}{cc}
c^{\prime} a & c^{\prime} a^{\prime} \\
b & 0 \\
0 & 1
\end{array}\right), \quad \beta_{m}=\left(\begin{array}{ccc}
1 & 0 & 0 \\
0 & -d & c a^{\prime}
\end{array}\right)
$$

we may extend $T$ to an exact tube of height $m+1$. By construction, the map

$$
\left(\begin{array}{c}
c^{\prime} a \\
b
\end{array}\right): Z \longrightarrow Z \oplus M
$$

is an $(M, N)$-monomorphism, and the square

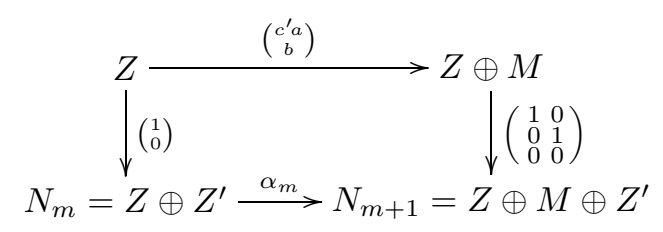

is exact with

$$
\left(\begin{array}{l}
1 \\
0
\end{array}\right)=\beta_{m} \circ\left(\begin{array}{l}
1 \\
0 \\
0
\end{array}\right)
$$

The result now follows from Remark 4.7.

Proposition 4.9. Let $T=\left(N_{i}, \alpha_{i}, \beta_{i}\right)$ be an exact tube of height $h+m$, for some $h \geq 1$ and $m \geq 1$. Suppose that

$$
N_{h+j+1} \underset{A}{\stackrel{\sim}{\longrightarrow}} N_{h+j} \oplus M
$$

for $j \in\{0, \ldots, m-1\}$. Then there is an $(M, N)$-monomorphism

$$
\left(\begin{array}{l}
f \\
g
\end{array}\right): N_{h+m-1} \longrightarrow N_{h+m-1} \oplus M
$$

such that the restrictions $T_{\leq m}$ and $\left(T_{f, g}\right)_{\leq m}$ are isomorphic.

Proof. We wish to choose

$$
\left(\begin{array}{l}
f \\
g
\end{array}\right)=\chi \circ \alpha_{h+m-1}
$$


for a suitable isomorphism $\chi: N_{h+m} \rightarrow N_{h+m-1} \oplus M$ and to apply Remark 4.7 to the diagram

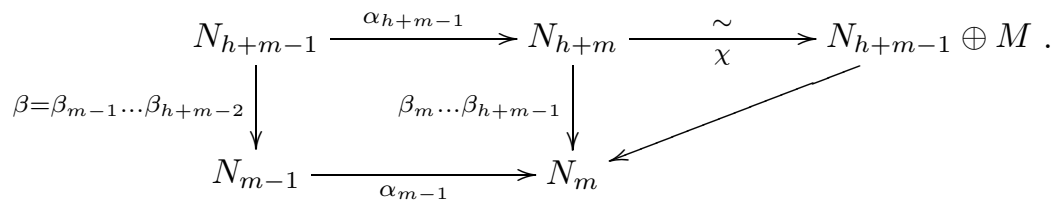

In order to do this, we only need to construct a section

$$
s: N_{h+m-1} \longrightarrow N_{h+m}
$$

satisfying

$$
\beta_{m-1} \beta_{m} \cdots \beta_{h+m-1} s=\beta \beta_{h+m-1} s=\beta .
$$

By our hypothesis, the square

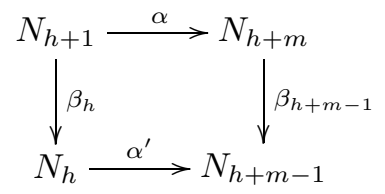

splits, where $\alpha=\alpha_{h+m-1} \ldots \alpha_{h+1}$ and $\alpha^{\prime}=\alpha_{h+m-2} \ldots \alpha_{h}$. Choose a maximal direct summand $A$ of $N_{h+m}$ for which $\left.\beta_{h+m-1}\right|_{A}$ is a section. Replacing $T$ by an isomorphic exact tube, we may assume that we have

$$
\begin{aligned}
\beta_{h+m-1} & =\left(\begin{array}{lll}
1 & 0 & 0 \\
0 & \gamma & \delta
\end{array}\right): A \oplus B \oplus M \longrightarrow A \oplus B, \\
\alpha^{\prime} & =\left(\begin{array}{ll}
\varepsilon & 0 \\
0 & 1
\end{array}\right): C \oplus B \longrightarrow A \oplus B
\end{aligned}
$$

for some maps $\gamma, \delta, \varepsilon$. Setting

$$
s=\left(\begin{array}{ll}
1 & 0 \\
0 & 1 \\
0 & 0
\end{array}\right): A \oplus B \longrightarrow A \oplus B \oplus M,
$$

we obtain

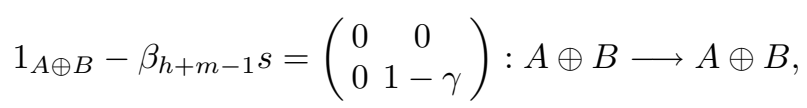

which factors through $\alpha^{\prime}$. But the sequence

$$
0 \longrightarrow N_{h} \stackrel{\alpha^{\prime}}{\longrightarrow} N_{h+m-1} \stackrel{\beta}{\longrightarrow} N_{m-1} \longrightarrow 0
$$

is exact, which implies $\beta=\beta \beta_{h+m-1} s$ as required. 


\section{Examples}

All our examples are representations of quivers with relations. Let $Q$ be a quiver with vertex set $Q_{0}=\{1, \ldots, n\}, I$ an admissible two-sided ideal in the quiver algebra $k Q, \underline{d}=\left(d_{1}, \ldots d_{n}\right)$ a vector in $\mathbb{N}^{n}$, and denote by

$$
\operatorname{Rep}(Q, I, \underline{d})
$$

the affine algebraic variety of representations $X$ of $(Q, I)$ with $X(i)=k^{d_{i}}, i \in Q_{0}$. The dimension vector of $X$ in $\operatorname{Rep}(Q, I, \underline{d})$ is $\underline{d}$. The group $G(\underline{d})=\prod_{i=1}^{n} G L\left(d_{i}\right)$ acts on $\operatorname{Rep}(Q, I, \underline{d})$ by

$$
(g \cdot X)(\alpha)=g_{j} \circ X(\alpha) \circ g_{i}^{-1}
$$

for an arrow $\alpha: i \rightarrow j$ and $g=\left(g_{1}, \ldots, g_{n}\right) \in G(\underline{d})$.

If we view $M, N$ in $\operatorname{Rep}(Q, I, \underline{d})$ as modules over $k Q / I$ of dimension $d=$ $\sum_{i=1}^{n} d_{i}$, then $M$ degenerates to $N$ if and only if the representation $N$ belongs to the closure of the orbit $G(\underline{d}) \cdot M$ of $M$ in $\operatorname{Rep}(Q, I, \underline{d})$ [1]. This allows us to work with the smaller group $G(\underline{d})$.

5.1. We begin with an example of a degeneration whose complexity is easy to compute: Choose a natural number $n \geq 2$ and let $\overrightarrow{A_{n}}$ be the equioriented quiver with underlying graph $A_{n}$ :

$$
\overrightarrow{A_{n}}=1 \stackrel{\gamma_{1}}{\longleftarrow} 2 \longleftarrow \cdots \longleftarrow n-1 \stackrel{\gamma_{n-1}}{\longleftarrow} n .
$$

Denote by $X_{i}$ the indecomposable representation of $\overrightarrow{A_{n}}$ given by

$$
\begin{gathered}
X_{i}(j)= \begin{cases}k & j \leq i, \\
0 & j>i,\end{cases} \\
X_{i}\left(\gamma_{j}\right)= \begin{cases}1 & j<i, \\
0 & j \geq i .\end{cases}
\end{gathered}
$$

Then $M=X_{n}$ has a filtration

$$
M=X_{n} \supset X_{n-1} \supset \cdots \supset X_{2} \supset X_{1},
$$

and it is well-known that $M$ degenerates to the associated graded module

$$
N=\bigoplus_{i=1}^{n} X_{i} / X_{i-1}
$$

where we set $X_{0}=0$. We wish to compute the complexity $\operatorname{cpl}(M, N)$, thereby 
showing again that $M$ actually degenerates to $N$. Set

$$
\begin{aligned}
& Z=\bigoplus_{i=1}^{n-1} X_{i} \\
& f=\left(\begin{array}{cccc}
0 & & & 0 \\
\iota_{2} & \ddots & & \\
& \ddots & \ddots & \\
& & \iota_{n-1} & 0
\end{array}\right): Z \longrightarrow Z \quad \text { and } \\
& g=\left(\begin{array}{lll}
0 \cdots 0 & \iota_{n}
\end{array}\right): Z \longrightarrow M=X_{n},
\end{aligned}
$$

where $\iota_{i}: X_{i-1} \rightarrow X_{i}$ is the inclusion. It is easy to check that $(f, g)^{t}$ is an $(M, N)$-monomorphism. Moreover, $f^{n-1}=0$, and thus

$$
\operatorname{cpl}(M, N) \leq n-1 \text {. }
$$

On the other hand, the Loewy lengths of $M$ and $N$ are $n$ and 1 , respectively, which implies

$$
\operatorname{cpl}(M, N) \geq \frac{\ell \ell(M)}{\ell \ell(N)}-1=n-1
$$

by Proposition 3.5. This example shows that there are degenerations of arbitrary complexity.

Note that for $n=4$ we obtain the following chain of degenerations:

$$
\begin{aligned}
M=k \stackrel{1}{\leftarrow} k \stackrel{1}{\leftarrow} k \stackrel{1}{\leftarrow} k & \leq_{\operatorname{deg}} P=k \stackrel{1}{\leftarrow} k \stackrel{0}{\leftarrow} k \stackrel{1}{\leftarrow} k \\
& \leq_{\operatorname{deg}} N=k \stackrel{0}{\leftarrow} k \stackrel{0}{\leftarrow} k \stackrel{0}{\leftarrow} k .
\end{aligned}
$$

The complexities are

$$
\begin{aligned}
& \operatorname{cpl}(M, P)=1=\operatorname{cpl}(P, N) \quad \text { and } \\
& \operatorname{cpl}(M, N)=3>\operatorname{cpl}(M, P)+\operatorname{cpl}(P, N) .
\end{aligned}
$$

Comparing with the example given in the introduction, we see that $\operatorname{cpl}(M, P)+$ $\operatorname{cpl}(P, N)$ can be either smaller or greater than $\operatorname{cpl}(M, N)$ for a chain

$$
M \leq_{\operatorname{deg}} P \leq_{\operatorname{deg}} N
$$

5.2. Next we give an example of a minimal degeneration of arbitrary complexity: Let $Q$ be the quiver

$$
Q=1 \stackrel{\alpha}{\longrightarrow} 2 \bigcap^{\beta}
$$

choose a natural number $n \geq 2$, and let $I$ be the ideal generated by $\beta^{n}$. Define $M$ and $N$ to be the representations of dimension vector $(1, n)$ given by

$$
M(\alpha)=e_{1}=\left(\begin{array}{c}
1 \\
0 \\
\vdots \\
\vdots
\end{array}\right), \quad N(\alpha)=e_{2}=\left(\begin{array}{c}
0 \\
1 \\
\vdots \\
0
\end{array}\right)
$$


and

$$
M(\beta)=N(\beta)=J_{n}, \text { respectively, }
$$

where $e_{1}, \ldots, e_{n}$ is the standard basis of $k^{n}$ and $J_{m}$ is the Jordan block

$$
J_{m}=\left(\begin{array}{cccc}
0 & & & 0 \\
1 & \ddots & & \\
& \ddots & \ddots & \\
0 & & 1 & 0
\end{array}\right)
$$

in $\mathbb{M}_{m}(k)$, for $m \in \mathbb{N}$.

Proposition 5.1. There is a degeneration $M \leq_{\operatorname{deg}} N$, which is minimal, and $\operatorname{cpl}(M, N)=n$.

Proof. Denote by $Z$ the indecomposable representation with dimension vector $(0, n)$, given by $Z(\beta)=J_{n}$, and let $(f, g)^{t}: Z \longrightarrow Z \oplus M$ be given by

$$
f=\left(0, J_{n}\right), \quad g=(0,1) .
$$

It is easy to see that $(f, g)^{t}$ is an $(M, N)$-monomorphism, so $M$ degenerates to $N$. Moreover, we have $f^{n}=0$, and therefore $\operatorname{cpl}(M, N) \leq n$. As

$$
\operatorname{dim} \operatorname{End} M=1 \text { and } \operatorname{dim} \operatorname{End} N=2,
$$

the orbit of $N$ has codimension 1 in the closure of the orbit of $M$, which implies that the degeneration is minimal.

Suppose $\operatorname{cpl}(M, N) \leq n-1$, and choose an $(M, N)$-tube $T=\left(N_{i}, \alpha_{i}, \beta_{i}\right)$ with $N_{n} \stackrel{\sim}{\sim} N_{n-1} \oplus M$. Let $\psi_{n}: N_{n} \rightarrow M$ be the surjection obtained from this decomposition.

Claim. For $i=1, \ldots, n$, there exists a surjection

$$
\psi_{i}: N_{i} \longrightarrow M^{(i)}
$$

where $M^{(i)}$ has dimension vector $(1, i)$ and is given by

$$
M^{(i)}(\alpha)=(1,0, \ldots, 0)^{t}, \quad M^{(i)}(\beta)=J_{i}
$$

Using the claim for $i=1$, we obtain a surjection $\psi_{1}: N_{1}=N \longrightarrow M^{(1)}$, which is impossible.

We prove the claim by descending induction on $i$. Observe that any map from $N$ to $M^{(i)}$ factors through the socle $\operatorname{soc} M^{(i)}$ and that $M^{(i)} / \operatorname{soc} M^{(i)} \stackrel{\sim}{\rightarrow} M^{(i-1)}$. Writing this factorization for $\psi_{i} \circ \alpha_{i-1} \cdots \alpha_{1}$, we obtain $\psi_{i-1}: N_{i-1} \rightarrow M^{(i-1)}$ from the following commutative diagram with exact rows: 


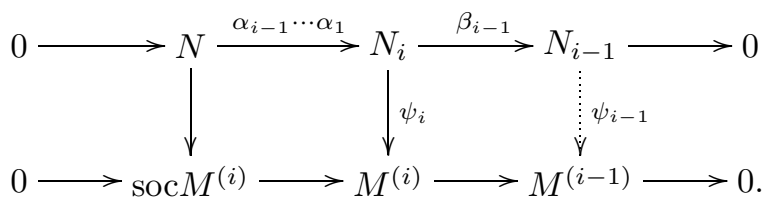

As $\psi_{i}$ is surjective, $\psi_{i-1}$ is as well.

A version of this argument implies the following result, which we will not use:

$$
\operatorname{cpl}\left(M, M_{i}\right)=\left[\frac{n-1}{i-1}\right]+1, \quad i \geq 2 .
$$

The representation $M_{i}$ is given by

$$
M_{i}(\alpha)=e_{i}, \quad M_{i}(\beta)=J_{n} .
$$

5.3. We now exhibit a degeneration $M \leq_{\operatorname{deg}} N$ of complexity 2 with the property that $f^{2} \neq 0$ for all $(M, N)$-monomorphisms $(f, g)^{t}$. Therefore the complexity can be strictly less than the "index of nilpotence of $M$ and $N$ "; i.e., the number

$$
\min \left\{r: f^{r}=0\right\},
$$

where the minimum is taken over all $(M, N)$-monomorphisms $(f, g)^{t}$. We stay with the same quiver $Q$, and we choose $I$ to be generated by $\beta^{3}$; i.e., we set $n=3$ in the preceding example. Note that $k Q / I$ is representation-finite: it admits 29 indecomposables [4].

We let $M$ and $N$ be given by

$$
M(\alpha)=e_{2}, \quad N(\alpha)=e_{3}, \quad M(\beta)=N(\beta)=J_{3},
$$

where $e_{1}, e_{2}, e_{3}$ is the standard basis of $k^{3}$. Choose

$$
\begin{gathered}
Z^{\prime}=0 \longrightarrow k^{3} J^{J_{3}} \\
f^{\prime}=\left(0, J_{3}\right): Z^{\prime} \longrightarrow Z^{\prime} \quad \text { and } \\
g^{\prime}=(0,1): Z^{\prime} \longrightarrow M .
\end{gathered}
$$

Then $\left(f^{\prime}, g^{\prime}\right)^{t}$ is an $(M, N)$-monomorphism. As $f^{\prime 2}$ factors through $g^{\prime}$, the cokernel $N_{3}$ of the map

$$
\varphi_{3}=\left(f^{\prime 3}, g^{\prime} f^{\prime 2}, g^{\prime} f^{\prime}, g^{\prime}\right)^{t}: Z^{\prime} \longrightarrow Z^{\prime} \oplus M^{3}
$$

used to define the tube $T_{f^{\prime}, g^{\prime}}=\left(N_{i}, \alpha_{i}, \beta_{i}\right)$ is isomorphic to the cokernel of

$$
\left(f^{\prime 2}, 0, g^{\prime} f^{\prime}, g^{\prime}\right)^{t}: Z^{\prime} \longrightarrow Z^{\prime} \oplus M^{3}
$$

and thus isomorphic to $M \oplus N_{2}$. By Lemma 3.3, we know that

$$
\operatorname{cpl}(M, N) \leq 2 .
$$


On the other hand, as $N$ is indecomposable, the complexity must exceed 1 , so

$$
\operatorname{cpl}(M, N)=2
$$

Claim. For any $(M, N)$-monomorphism

$$
(f, g)^{t}: Z \longrightarrow Z \oplus M
$$

we have $f^{2} \neq 0$.

First we show:

Lemma 5.2. For any $(M, N)$-monomorphism

$$
(f, g)^{t}: Z \longrightarrow Z \oplus M
$$

$Z^{\prime}$ is a direct summand of $Z$.

Proof. Consider the exact sequences

$$
\Sigma^{\prime}: 0 \longrightarrow Z^{\prime} \stackrel{\left(\begin{array}{l}
f^{\prime} \\
g^{\prime}
\end{array}\right)}{\longrightarrow} Z^{\prime} \oplus M \stackrel{\left(k^{\prime}, l^{\prime}\right)}{\longrightarrow} N \longrightarrow 0
$$

and

$$
\Sigma: 0 \longrightarrow Z \stackrel{\left(\begin{array}{l}
f \\
g
\end{array}\right)}{\longrightarrow} Z \oplus M \stackrel{(k, l)}{\longrightarrow} N \longrightarrow 0 .
$$

It is easy to check that

$$
\begin{aligned}
\operatorname{dim} \operatorname{Hom}\left(Z^{\prime}, M\right) & =\operatorname{dim} \operatorname{Hom}\left(Z^{\prime}, N\right)=3, \\
\operatorname{dim} \operatorname{End} M & =\operatorname{dim} \operatorname{Hom}(M, N)=2 .
\end{aligned}
$$

Therefore the sequence of vector spaces

$$
0 \longrightarrow \operatorname{Hom}\left(Z^{\prime} \oplus M, Z\right) \longrightarrow \operatorname{Hom}\left(Z^{\prime} \oplus M, Z \oplus M\right) \longrightarrow \operatorname{Hom}\left(Z^{\prime} \oplus M, N\right) \longrightarrow 0
$$

obtained from mapping $Z^{\prime} \oplus M$ into $\Sigma$ is exact. In particular, $\left(k^{\prime}, l^{\prime}\right): Z^{\prime} \oplus M \rightarrow N$ factors through $(k, l): Z \oplus M \rightarrow N$, and hence we have the following commutative diagram (Figure 3 ) with exact rows and columns. 


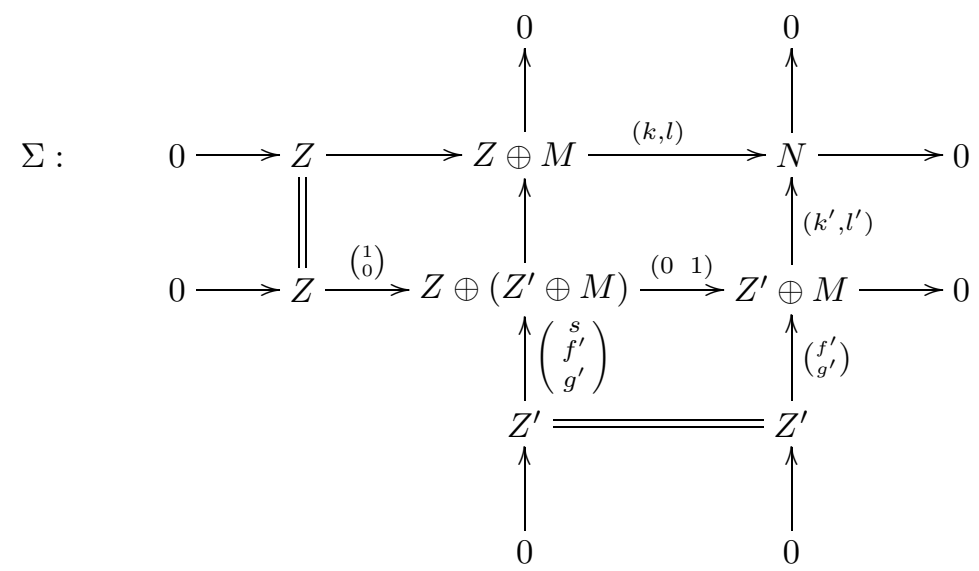

Figure 3

So the middle column splits as well, and since by construction $f^{\prime}, g^{\prime}$ lie in the radical,

$$
s: Z^{\prime} \longrightarrow Z
$$

must be a section.

Let $(f, g)^{t}: Z \rightarrow Z \oplus M$ be an $(M, N)$-monomorphism, suppose $f^{2}=0$, and consider the commutative diagram (Figure 4 ) with exact rows and columns.

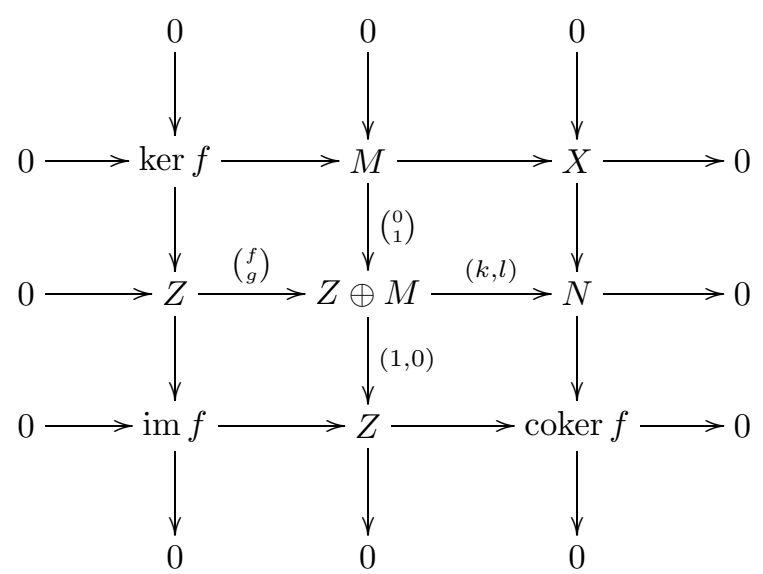

Figure 4

Then $X$ must be a quotient of $M$ and a submodule of $N$, which is possible in 
exactly two ways:

(i) $X=k \stackrel{\left(\begin{array}{l}0 \\ 1\end{array}\right)}{\rightarrow}>k^{2} \bigcirc J_{2}$

(ii) $X=0 \longrightarrow k \bigcirc 0$

In the first case, we have

$$
\text { ker } f=0 \longrightarrow k \bigcap 0,
$$

and our assumption $f^{2}=0$ implies that $\operatorname{dim} Z(2) \leq 2$. But then $Z$ cannot contain $Z^{\prime}$ as a direct summand.

In the second case, we see that

$$
\operatorname{ker} f=k \stackrel{\left(\begin{array}{l}
1 \\
0
\end{array}\right)}{\rightarrow} k^{2} \bigcirc J_{2} .
$$

Now $f^{2}=0$ implies that $\operatorname{dim} Z(2) \leq 4$. But then necessarily $Z(\beta \alpha)=0$, since $Z^{\prime}$ must be a direct summand of $Z$, and $Z$ cannot contain $\operatorname{ker} f$ as a submodule.

5.4. As our last example, we find a degeneration $M \leq_{\operatorname{deg}} N$ of complexity 2 for which there exists an exact sequence

$$
\Sigma: 0 \longrightarrow N \stackrel{\alpha_{1}=\left(\begin{array}{l}
f \\
g
\end{array}\right)}{\longrightarrow} N \oplus M \stackrel{\beta_{1}=(f,-l)}{\longrightarrow} N \longrightarrow 0 .
$$

So we have an exact tube

$$
T=\left(N_{1}=N, N_{2}=N \oplus M, \alpha_{1}, \beta_{1}\right)
$$

of height 2. If this tube were the restriction of an (infinite) exact tube, the complexity $\operatorname{cpl}(M, N)$ would have to equal 1 . So the number $2 h+1$ in condition (iii) of our main theorem cannot be replaced by $2 h$.

Choose $A=k[\alpha, \beta] /\left(\alpha^{2}, \beta^{2}\right)$, let $M$ and $N$ be 4-dimensional with

$$
M(\alpha)=N(\alpha)=\left(\begin{array}{llll}
0 & 0 & 0 & 0 \\
0 & 0 & 0 & 0 \\
1 & 0 & 0 & 0 \\
0 & 1 & 0 & 0
\end{array}\right), M(\beta)=\left(\begin{array}{llll}
0 & 0 & 0 & 0 \\
1 & 0 & 0 & 0 \\
0 & 0 & 0 & 0 \\
0 & 0 & 1 & 0
\end{array}\right), N(\beta)=\left(\begin{array}{llll}
0 & 0 & 0 & 0 \\
0 & 0 & 0 & 0 \\
0 & 0 & 0 & 0 \\
1 & 0 & 0 & 0
\end{array}\right),
$$

and set

$$
f=\left(\begin{array}{llll}
0 & 0 & 0 & 0 \\
1 & 0 & 0 & 0 \\
0 & 1 & 0 & 0 \\
0 & 0 & 1 & 0
\end{array}\right), g=\left(\begin{array}{llll}
0 & 0 & 0 & 0 \\
0 & 1 & 0 & 0 \\
1 & 0 & 0 & 0 \\
0 & 0 & 0 & 1
\end{array}\right), l=\left(\begin{array}{llll}
1 & 0 & 0 & 0 \\
0 & 0 & 0 & 0 \\
0 & 0 & 1 & 0 \\
0 & 1 & 0 & 0
\end{array}\right) .
$$

It is easy to check that the sequence $\Sigma$ obtained from these choices is exact. So $M$ degenerates to $N$. As $N$ is indecomposable and $f^{2}$ factors through $g$, the same argument as in Section 5.3 implies that $\operatorname{cpl}(M, N)=2$.

This example has another surprising feature: For any degeneration $M \leq_{\operatorname{deg}} N$ we obtain

$$
\operatorname{cpl}\left(M^{r}, N^{r}\right) \leq \operatorname{cpl}(M, N), \quad r \geq 1,
$$


by taking for $M^{r} \leq_{\operatorname{deg}} N^{r}$ the direct sum of $r$ copies of an $(M, N)$-tube of minimal complexity. In our example, we have

$$
\operatorname{cpl}\left(M^{2}, N^{2}\right)=1<\operatorname{cpl}(M, N)=2 .
$$

Indeed, $M^{2}$ is a projective cover for $N$, and the kernel of an epimorphism $M^{2} \rightarrow N$ is $N$ again. So there is an exact sequence

$$
0 \longrightarrow N \longrightarrow M^{2} \longrightarrow N \longrightarrow 0
$$

Acknowledgments. We would like to thank the Schweizerischer Nationalfonds for supporting the first and the third author. This article was written while the third author was visiting the University of Berne; he also gratefully acknowledges support from Polish Scientific Grant KBN No. 2 PO3A 012 14. This paper is part of the first author's thesis.

\section{References}

[1] K. Bongartz, A geometric version of the Morita equivalence, J. Algebra 139 (1991), 159171.

[2] F. Grunewald and J. O'Halloran, A characterization of orbit closure and applications, $J$. Algebra 116 (1988), 163-175.

[3] H. Kraft, Geometrische Methoden in der Invariantentheorie, Vieweg, 1984.

[4] Ch. Riedtmann, Algebren, Darstellungsköcher und zurück, Comment. Math. Helv. 55 (1980), 199-224.

[5] Ch. Riedtmann, Degenerations for representations of quivers with relations, Ann. Sci. École Normal Sup. 19 (1986), 275-301.

[6] G. Zwara, A degeneration-like order for modules, Arch. Math. 71 (1998), 437-444.

[7] G. Zwara, Degenerations of modules are given by extensions, Compositio Math 121 (2000), 205-218.

Robert Aehle

Mathematisches Institut

Universität Bern

Sidlerstrasse 5

3012 Bern

Switzerland

e-mail: robert.aehle@math-stat.unibe.ch

Grzegorz Zwara

Faculty of Mathematics and Informatics

Nicholas Copernicus University

Chopina 12/18

87-100 Toruń

Poland

e-mail: gzwara@mat.uni.torun.pl
Christine Riedtmann

Mathematisches Institut

Universität Bern

Sidlerstrasse 5

3012 Bern

Switzerland

e-mail: christine.riedtmann@math-stat.unibe.ch

(Received: January 30, 2001) 